Annales Geophysicae (2001) 19: 883-888 C European Geophysical Society 2001

\title{
Anisotropy of Doppler spectral parameters in the VHF radar observations at MU and White Sands
}

\author{
G. D. Nastrom ${ }^{1, *}$ and T. Tsuda ${ }^{1}$ \\ ${ }^{1}$ Radio Science Center for Space and Atmosphere, Kyoto University, Kyoto 611, Japan \\ *Permanent address: Department of Earth Sciences, St. Cloud State University, St. Cloud, MN 56379, USA
}

Received: 12 September 2000 - Revised: 7 March 2001 - Accepted: 22 March 2001

\begin{abstract}
Significant differences are found between the mean spectral widths from beams in the meridional plane and in the zonal plane at both the MU and the White Sands VHF radars. The spectral width in the beam directed perpendicular to the prevailing wind is greater than that in the beam parallel to the wind. The magnitudes of the differences in spectral width show a linear relationship with wind speed, with the greatest differences at the greatest wind speeds. The differences in spectral width show a positive correlation with the differences in backscattered power. The anisotropy in backscattered power is well-known and is usually attributed to aspect sensitivity effects. However, the anisotropy in spectral width does not appear to be due to the same mechanism, and while several hypotheses to account for this are considered, including finite range-volume effects, effects from the tilting of isentropic layers, and anisotropic turbulence effects, it is seen that each of these suggestions has its shortcomings.
\end{abstract}

Key words. Meteorology and atmospheric dynamics (turbulence; instruments and techniques)

\section{Introduction}

The azimuthal anisotropy of the backscattered power received on oblique beams of mesosphere-stratosphere-troposphere (MST) radars from the troposphere and stratosphere has been discussed in many studies (e.g. Hocking, 1987; Hocking et al., 1990; Hooper and Thomas, 1995; Tsuda et al., 1986, 1997a,b; Worthington and Thomas, 1997; Worthington et al., 1999, 2000). The anisotropy in backscattered power is usually attributed to aspect sensitivity effects associated with the tilting of aspect sensitive surfaces by gravity waves and, as discussed by Worthington and Thomas (1997), vertical wind shears.

Correspondence to: G. D. Nastrom

(nastrom@stcloudstate.edu)
In contrast, there have been very few studies of the azimuthal anisotropy of Doppler spectral width. Nastrom and Eaton (1995) noted that the mean values of spectral width and backscattered power at the White Sands (WS) VHF radar in New Mexico are larger in the north-south beam than in the east-west beam. A brief experiment reported by Nastrom and Eaton (1997) could not determine if the differences were of instrumental or atmospheric origin. The present study reports the observed azimuthal anisotropy of the spectral widths, which were determined by using the long-term data bases available from WS and from the Middle and Upper Atmosphere (MU) radar located near Shigaraki, Japan. In an effort to understand the origin of these anisotropies, their relationships to other variables measured by the radars will also be discussed.

\section{Data}

WS and MU are both very large VHF radars with one-way $3 \mathrm{~dB}$ beamwidths $(2 \theta)$ of $2.9^{\circ}$ and $3.6^{\circ}$, respectively. They both use 150-m range gates $(\Delta R)$ over the altitude range of approximately $5-20 \mathrm{~km}$. MU data are for five beam directions (vertical and at $10^{\circ}$ zenith angle $(\alpha)$ toward the four cardinal directions) and WS has three beams (vertical and at $\alpha=15^{\circ}$ toward the north and toward the east). Further technical details of the MU and WS radars are given by Fukao et al. (1990) and Nastrom and Eaton (1993), respectively.

MU and WS are at similar latitudes $\left(34.9^{\circ} \mathrm{N}\right.$ and $32.4^{\circ} \mathrm{N}$, respectively), although other aspects of their locations and operating environments are quite different. For example, maximum monthly mean zonal wind speeds at $\mathrm{MU}$ range from less than $10 \mathrm{~m} / \mathrm{s}$ during August to over $60 \mathrm{~m} / \mathrm{s}$ during December, January, and February, while at WS, the range is from about $5 \mathrm{~m} / \mathrm{s}$ during August to about $35 \mathrm{~m} / \mathrm{s}$ during December (Nastrom and Tsuda, 2000). Meridional winds are usually relatively light at both sites and, consequently, the flow tends to be from the west.

Hourly profiles of the means, medians, and standard devia- 

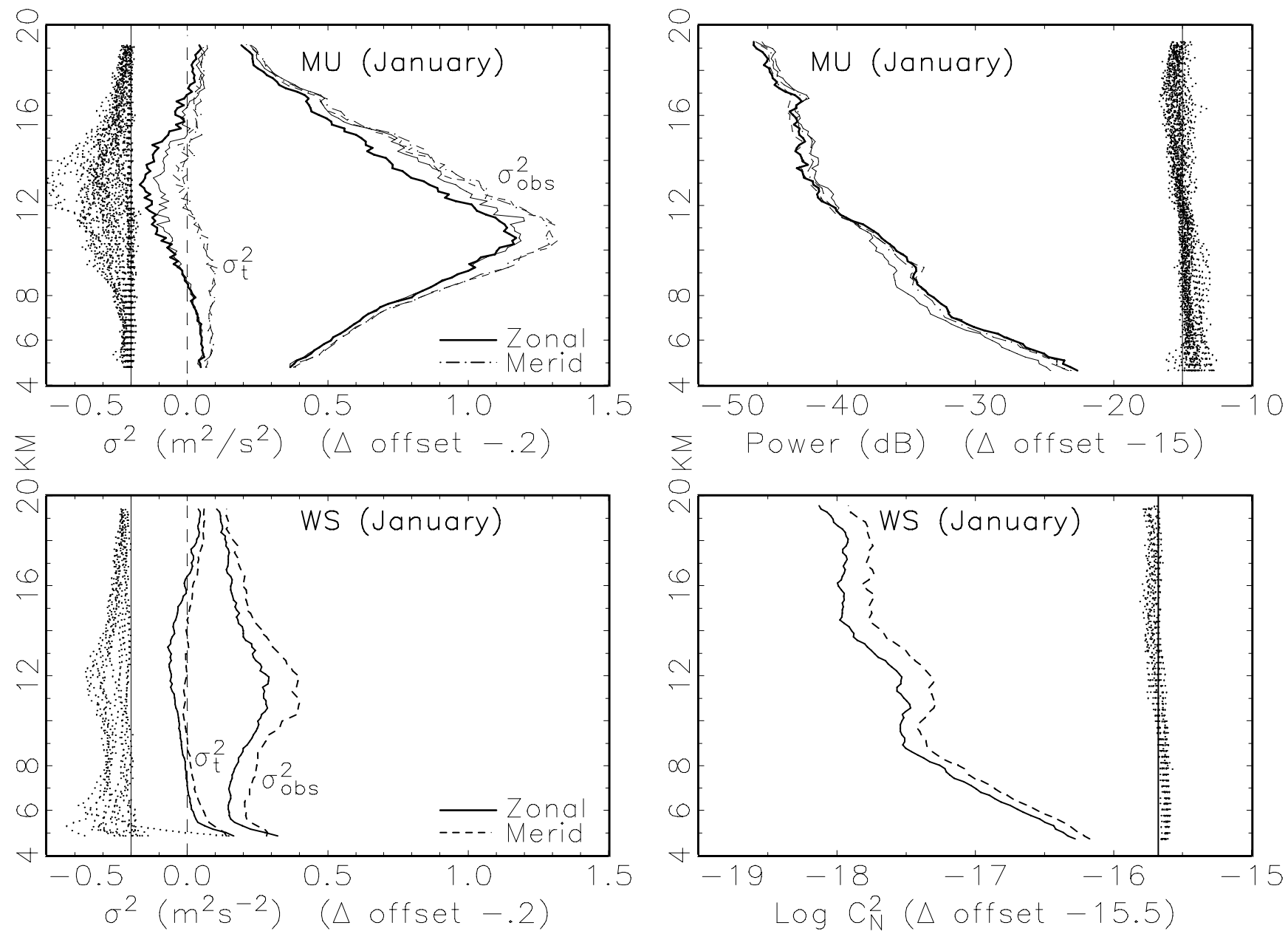

Fig. 1. Vertical profiles of the median spectral width (left) and returned power (right) for MU (upper) and WS (lower) during January. Solid (dashed) lines are for beams in the zonal (meridional) plane. Dotted lines show the differences in the values between the zonal and meridional beams.

tions of the radial velocity, spectral width, and backscattered power were computed for each radar beam at each site. At MU, data were available for 12363 hours from the regular observation periods (about 100 hours per month) from 1986 through 1996. At WS, profiles for 25656 hours were available, taken during the period January 1991 through September 1996 .

The backscattered power at MU was reported in $\mathrm{dB}$ relative to an arbitrary reference; for use here, it has been multiplied by the square of the range. The backscattered power at WS was reported as $C_{N}^{2}$. At large $\alpha$ the backscattered power at VHF is from Bragg scattering, while at small $\alpha$, other processes lead to aspect sensitivity effects (e.g. Chau et al., 2000). Studies of aspect sensitivity (e.g. Tsuda et al., 1986, 1997a) indicate that the backscattered power decreases sharply with $\alpha$ out to about $10^{\circ}$ (although in some cases, aspect sensitivity may extend to higher $\alpha$ ). As mentioned above, tilting of aspect sensitive surfaces by gravity waves or other processes can lead to apparent azimuthal anisotropy of backscattered power.

The observed spectral width $\left(\sigma_{\text {obs }}^{2}\right.$; the $3 \mathrm{~dB}$ half-width squared divided by $2 \ln 2$ ) contains contributions due to atmospheric turbulence within the sample volume $\left(\sigma_{\mathrm{t}}^{2}\right)$ and other effects due to beam-, shear-, and wave-broadening $\left(\sigma_{\text {corr }}^{2}\right)$. These contributions are additive (Gossard,1990),

$\sigma_{\mathrm{obs}}^{2}=\sigma_{\mathrm{t}}^{2}+\sigma_{\mathrm{corr}}^{2}$

Nastrom (1997) found that the beam- and shear-broadening contributions to $\sigma_{\text {corr }}^{2}$ contain a cross-term, i.e.

$$
\begin{aligned}
& \sigma_{\text {beam }+ \text { shear }}^{2}=\frac{\theta^{2}}{3}\left\{\left(u^{2} C^{2}+v^{2}\right)-2 S^{2}\right. \text { CRuu } \\
& \left.\quad+u_{z}^{2}\left(R^{2} S^{4}+\frac{\Delta R^{2}}{12} \cos ^{4} \alpha\right)\right\}+u_{z}^{2} \frac{\Delta R^{2}}{48} \sin ^{2} 2 \alpha
\end{aligned}
$$

where $C=\cos \alpha, S=\sin \alpha, R$ is range, $u$ is the speed of the wind in the plane of the beam, $v$ is that in the plane perpendicular to the beam, and $u_{z}$ is the vertical gradient of $u$. The first term inside the braces, called the beam-broadening term, is usually much larger than the others, although in strong shear conditions, the second term, called the cross-term, can 

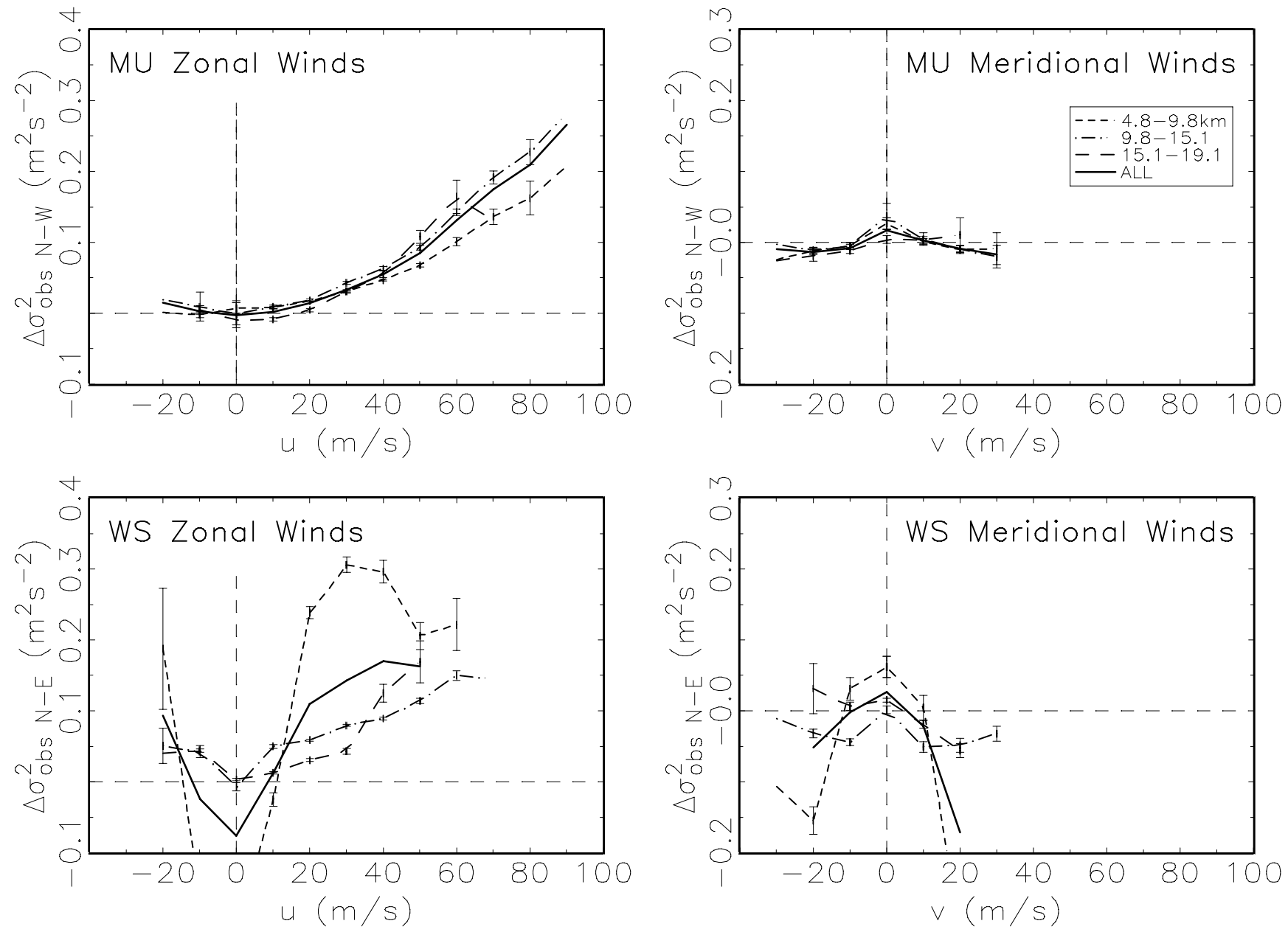

Fig. 2. Differences in $\sigma_{\mathrm{obs}}^{2}$ values between the meridional and zonal beams for the prevailing zonal (meridional) flow in the left (right) panels for MU (upper) and WS (lower). See text.

also be significant. The terms in $u_{z}^{2}$ are nearly always negligible. The contribution due to wave-broadening is also nearly always negligible (Nastrom and Eaton, 1997).

\section{Results}

Figure 1 shows the profiles of $\sigma_{\mathrm{obs}}^{2}$ for January to illustrate the results; during other months (not shown), the results are similar during winter and decrease during summer, with minimum values near zero during August. In Fig. 1, the bold (light) solid line at MU is for the beam directed toward the west (east). The median $\sigma_{\text {obs }}^{2}$ is greater in the zonal beams than in the meridional beams at both MU and WS. (The medians for the east and west beam at MU are different above approximately $12 \mathrm{~km}$; the origin of this difference is unknown and in this study we will focus on the differences between the west beam and the meridional beams). The magnitudes of the differences between $\sigma_{\mathrm{obs}}^{2}$ in the meridional plane and the zonal plane are about the same at MU (north beam minus west beam at MU) and WS, ranging up to about $0.2 \mathrm{~m}^{2} \mathrm{~s}^{-2}$. The differences $\left(\Delta \sigma_{\mathrm{obs}}^{2}\right)$ for all twelve calendar months are shown in Fig. 1 by dotted lines; for clarity, they have been offset toward the left by $0.2 \mathrm{~m}^{2} \mathrm{~s}^{-2}$. The largest (smallest) $\Delta \sigma_{\text {obs }}^{2}$ are for winter (summer) months.

The shapes of the profiles of $\sigma_{\text {obs }}^{2}$ resemble the shapes of the profiles of wind speed at both sites. Indeed, Eq. (2) shows that the beam-broadening contribution to $\sigma_{\text {obs }}^{2}$ is very closely related to wind speed. The profiles of $\sigma_{\mathrm{t}}^{2}$ in Fig. 1 were obtained by applying the standard corrections for beam, shear, and wave broadening to $\sigma_{\mathrm{obs}}^{2}$ (following Nastrom and Eaton, 1997). Some of the values are negative when wind speeds are large, as discussed by Fukao et al. (1994). While the shapes of the $\sigma_{\mathrm{t}}^{2}$ profiles are different from those for $\sigma_{\mathrm{obs}}^{2}$, the differences between the zonal and meridional directions are about the same, i.e. applying $\sigma_{\text {corr }}^{2}$ does not significantly diminish the azimuthal anisotropy.

In Fig. 1, the largest differences in the values between the zonal and meridional beams are found near $11 \mathrm{~km}$, near the level of maximum wind speed. Figure 2 further illustrates the relationship between the magnitudes of the anisotropies $\left(\Delta \sigma_{\mathrm{obs}}^{2}\right.$, north minus west at MU) and wind speed. Mean values of $\Delta \sigma_{\mathrm{obs}}^{2}$ are plotted for $10 \mathrm{~ms}^{-1}$ wind-speed bins. The 
Table 1. Coefficients of correlation of $\Delta \sigma_{\mathrm{obs}}^{2}$ and $\Delta \sigma_{\mathrm{t}}^{2}$ (meridional plane minus zonal plane) with other variables measured by the VHF radars at MU and WS. Correlations of magnitude $\geq 0.2$ are flagged with a star

\begin{tabular}{lllllll}
\hline \multicolumn{5}{c}{ WS } & & \multicolumn{3}{c}{ MU } \\
\hline & $7 \mathrm{~km}$ & $12 \mathrm{~km}$ & $17 \mathrm{~km}$ & $7 \mathrm{~km}$ & $12 \mathrm{~km}$ & $17 \mathrm{~km}$ \\
\hline$N_{0}$ & 14033 & 11793 & 13499 & 11073 & 9330 & 9325 \\
\hline Correlation with $\Delta \sigma_{\text {obs }}^{2}$ & & & & & & \\
\hline Wind speed & 0.16 & 0.12 & 0.07 & $0.25 *$ & $0.25 *$ & 0.11 \\
$u$ & $0.25 *$ & 0.17 & 0.09 & $0.26 *$ & $0.26 *$ & 0.10 \\
$v$ & 0.04 & 0.08 & 0.05 & -0.04 & 0.05 & 0.15 \\
$d u / d z$ & 0.03 & $0.20 *$ & $0.25 *$ & $0.32 *$ & $0.22 *$ & $0.37 *$ \\
$d v / d z$ & 0.01 & 0.09 & 0.01 & 0.02 & $-0.21 *$ & -0.07 \\
\hline Correlation with $\Delta \sigma_{\mathrm{t}}^{2}$ & & & & & & \\
\hline Wind speed & 0.14 & 0.13 & 0.12 & $0.26 *$ & $0.21 *$ & 0.11 \\
$u$ & $0.23 *$ & 0.19 & 0.15 & $0.27 *$ & $0.22 *$ & 0.10 \\
$v$ & 0.03 & 0.03 & -0.10 & -0.03 & 0.04 & 0.14 \\
$d u / d z$ & -0.00 & $-0.31 *$ & $-0.38 *$ & $0.38 *$ & $0.28 *$ & $0.38 *$ \\
$d v / d z$ & 0.01 & 0.05 & 0.09 & 0.04 & -0.01 & -0.04 \\
$\Delta$ power & 0.15 & $0.34 *$ & $0.39 *$ & 0.04 & 0.17 & $0.21 *$ \\
power $v$ & 0.08 & 0.16 & 0.12 & -0.04 & 0.02 & -0.03 \\
$\sigma_{w}$ & 0.03 & 0.00 & 0.02 & 0.03 & 0.00 & 0.02 \\
$\sigma_{t}^{2}-v$ & $0.68 *$ & $0.55 *$ & $0.24 *$ & $0.39 *$ & $0.32 *$ & 0.14 \\
\hline
\end{tabular}

abscissa in the left-hand (right-hand) panels is the zonal $(u)$ (meridional $(v)$ ) wind speed and only observations with wind directions within $30^{\circ}$ of east-west (north-south) were used. Data were sorted into three altitude groups, representing the troposphere $(4.8-9.8 \mathrm{~km})$, near the level of maximum winds $(9.8-15.1 \mathrm{~km})$, and the lower stratosphere $(15.1-19.1 \mathrm{~km})$. Error bars extend $\pm 2 \sigma / \sqrt{N_{0}}$ (where $\sigma$ is the standard deviation of the $N_{0}$ data points in each group) and thus represent the $95 \%$ confidence interval for the mean of each group.

The curves in Fig. 2 are all concave in the left-hand panels and convex in the right-hand panels, indicating that $\sigma_{\text {obs }}^{2}$ in the beam perpendicular to the wind is greater than that in the beam parallel to the wind. The corresponding curves for $\Delta \sigma_{t}^{2}$ as a function of wind speeds (not shown) have nearly identical shapes since the main correction term is due to beam broadening, which is about the same magnitude for both zonal and meridional planes.

Table 1 gives the correlation coefficients of hourly values of $\Delta \sigma_{\text {obs }}^{2}$ with wind speed, $u, v$, and the vertical shears of the wind, at three representative levels. The correlation coefficients of $\Delta \sigma_{t}^{2}$ with the same variables and with the anisotropy of backscattered power $\left(C_{n}^{2}\right.$ at WS), backscattered power in the meridional beam, the hourly standard deviation of the vertical velocity $\left(\sigma_{w}\right)$, and the value of $\sigma_{\mathrm{t}}^{2}$ in the meridional beam are also given. In order to focus attention on wind speed and wind shear (i.e. baroclinic) effects rather than possible convective effects, only data for September through May were used in Table 1. Although all correlation coefficients greater than approximately 0.04 are statistically significant due to the large number of data pairs used here, we have chosen to flag only magnitudes $\geq 0.2$ in Table 1 .

At 7 and $12 \mathrm{~km}$, the correlations are approximately the same for the total wind speed and for the zonal component, and correlations with $v$ are $\leq 0.15$ at all heights. Correlations with $d u / d z$ are all large, except at $7 \mathrm{~km}$ at WS. Most correlations with $d v / d z$ are $<0.2$.

The correlations with $\Delta$ power seen in Table 1 are positive and increase with altitude at both WS and MU. Because the anisotropy in backscattered power is often attributed to gravity wave activity (e.g. Hocking, 1987; Tsuda et al., 1997b; Worthington et al., 1999), this pattern of correlation coefficients might suggest that there is a relationship between $\Delta \sigma_{\mathrm{t}}^{2}$ and the intensity of gravity wave activity. However, the correlation of $\Delta \sigma_{\mathrm{t}}^{2}$ with $\sigma_{w}$ (often used as an indicator of gravity wave intensity) is insignificant.

The correlation coefficents of $\Delta \sigma_{\mathrm{t}}^{2}$ with $\sigma_{\mathrm{t}}^{2}-v$ are large and positive at nearly all levels. These results imply that $\Delta \sigma_{\mathrm{t}}^{2}$ becomes larger as $\sigma_{\mathrm{t}}^{2}$ becomes larger.

Returning to Fig. 1, the right-hand panels show the vertical profiles of the median power (multiplied by the rangesquared) at MU and of $C_{N}^{2}$ at WS. At MU, the power in the west beam (the bold solid line) is generally greater (less) than that in the other beams in the troposphere (stratosphere). Most of the monthly mean differences relative to the west beam, shown by dotted lines offset $-15 \mathrm{~dB}$, fall to the right (left) of the vertical reference line below (above) approximately $11 \mathrm{~km}$. At WS, there is a bias in $\log C_{N}^{2}$ between the meridional and the zonal beams; the mean magnitude of the bias over all heights is 0.175 . It is not known if the source of the mean bias is instrumental. However, note that the dif- 
ferences between the zonal and meridional beams' values, shown by the dotted lines (plotted offset by -15.5 ), have a pattern in the vertical similar to that seen at MU, i.e. they are to the right (left) of the reference line entered at -15.675 below (above) approximately $11 \mathrm{~km}$. The anisotropy in the backscattered power as a function of azimuth, as seen here, is consistent with that seen in past studies. Several studies have shown that the changes in anisotropy in backscattered power are largely due to aspect sensitivity effects associated with the jet stream arising from gravity waves (e.g. Hocking, 1987; Tsuda et al., 1997b; Worthington et al., 1999) or vertical wind shear effects (Worthington and Thomas, 1997).

\section{Discussion}

Several hypotheses for the azimuthal anisotropy of spectral width and its relationship to wind speed have been considered, including finite range-volume effects, tilting of isentropic layers, anisotropic turbulence effects, and anisotropic beam-broadening effects. None of these suggestions, however, can fully explain the observations.

Finite range-volume effects (Fukao et al., 1988) do not apply below the altitude given by $\Delta R(2 \theta \sin \alpha)^{-1}$, which is $11.1 \mathrm{~km}$ at $\mathrm{WS}$ and $13.8 \mathrm{~km}$ at MU. The profiles of $\Delta \sigma_{\mathrm{obs}}^{2}$ in Fig. 1 are much different from zero below these levels.

Worthington et al. (1999) sketch a model where gravity waves or Kelvin-Helmholz instabilities (KHI) cause anisotropy in the returned power by tilting isentropic aspectsenisitve surfaces. Specular echoes have relatively narrow spectral widths compared to turbulent echoes, and if there are a large number of specular echoes in one direction, as compared to another, there may be an associated anisotropy of the spectral widths. However, although the differences in the returned power profiles in Fig. 1 are consistent with the aspect sensitive surfaces being tilted one way below the wind maximum and the other way above it, the differences in spectral width are in the same sense at all heights.

Hocking and Hamza (1997) present a model for anisotropic turbulence (their Eq. 6), in which the greatest anisotropy occurs when the turbulence is weak. In the present results, there is a strong positive correlation (Table 1) between $\Delta \sigma_{t}^{2}$ and $\sigma_{\mathrm{t}}^{2}$, indicating that the largest values of $\Delta \sigma_{\mathrm{t}}^{2}$ are associated with largest $\sigma_{\mathrm{t}}^{2}$.

The formula for the beam-broadening term when written for a beam at azimuthal angle $\chi$ relative to the wind is $\sigma_{B}^{2}=$ $\left(\sin ^{2} \chi+\cos ^{2} \chi \cos ^{2} \alpha\right) V^{2} \theta^{2} / 3$, where $V$ is wind speed. While this formula anticipates some azimuthal anisotropy in the correction for beam-broadening, the magnitude of this anisotropy ranges to only $3 \%$ for $\alpha=10^{\circ}$ at $\mathrm{MU}$, and $7 \%$ for $\alpha=15^{\circ}$ at WS, which is much smaller than the observed anisotropy (there is another difference among the corrections to the cross- and along-wind beams according to Eq. (2), but it is less than $1 \%$ of the beam broadening term and thus is negligible).

Finally, it is possible that there is another (as yet unknown) correction term that needs to be applied to the spectral widths which is different for the beams parallel and perpendicular to the wind. This suggestion is currently being investigated using observations from MU and the results will be reported in the future.

Acknowledgements. GDN was partially supported by the Air Force Office of Scientific Research.

Topical Editor D. Murtagh thanks a referee for his help in evaluating this paper.

\section{References}

Chau, J. L., Doviak, R. J., Muschinski, A., and Holloway, C. L., Tropospheric measurements of turbulence and characteristics of Bragg scatter using the Jicamarca VHF radar, Radio Sci., 35, 179-194, 2000.

Fukao, S., Sato, T., May, P. T., Tsuda, T., Kato, S., Inaba, M., and Kimura, I., A systematic error in MST/ST radar wind measurement induced by a finite range volume effect 1 , Observational results, Radio Sci., 23, 59-73, 1988.

Fukao, S., Sato, T., Tsuda, T., Yamamoto, M., Yamanaka, M., and Kato, S., MU radar: New capabilities and system calibrations, Radio Sci., 25, 477-485, 1990.

Fukao, S., Yamanaka, M. D., Ao, N., Hocking, W. K., Sato, T., Yamamoto, M., Nakamura, T., Tsuda, T., and Kato, S., Seasonal variability of vertical eddy diffusivity in the middle atmosphere 1, Three-year observations by the middle and upper atmosphere radar, J. Geophys. Res., 99, 18973-18987, 1994.

Gossard, E. E., Radar research on the atmospheric boundary layer, Radar in Meteorology, Ed. D. Atlas, Am. Meteor. Soc., Boston, Chapt. 27a., 477-527, 1990

Hocking, W., Reduction of the effects of non-stationarity in studies of amplitude statistics of radio wave backscatter, J. Atmos. Terr. Phys., 49, 1119-1131, 1987.

Hocking, W. K., Fukao, S., Tsuda, T., Yamamoto, M., Sato, T., and Kato, S., Aspect sensitivity of stratospheric VHF radio wave scatterers, particularly above $15-\mathrm{km}$ altitude, Radio Sci., 25, 613-627, 1990.

Hocking, W. K. and Hamza, A. M., A quantitative measure of the degree of anisotropy of turbulence in terms of atmospheric parameters, with particular relevance to radar studies, J. Atmos. Solar-Terr. Phys., 59, 1011-1020, 1997.

Hooper, D. and Thomas, L., Aspect sensitivity of VHF scatterers in the troposphere and stratosphere from comparisons of powers in off-vertical beams, J. Atmos. Solar-Terr. Phys, 57, 655-663, 1995.

Nastrom, G. D., Doppler radar spectral width broadening due to beamwidth and wind shear, Ann. Geophysicae, 15, 786-796, 1997.

Nastrom, G. D. and Eaton, F. D., The coupling of gravity waves and turbulence at White Sands, New Mexico, from VHF radar observations, J. Appl. Meteor., 32, 81-87, 1993.

Nastrom, G. D. and Eaton, F. D., Variations of winds and turbulence seen by the $50 \mathrm{MHz}$ radar at White Sands Missile Range, NM, J. Appl. Meteor., 34, 2135-2148, 1995.

Nastrom, G. D. and Eaton, F. D., Turbulence eddy dissipation rates from radar observations at 5-20 km at White Sands Missile Range, New Mexico, J. Geophys. Res., 102, 19495-19506, 1997.

Nastrom,, G. D. and Tsuda, T., Comparisons of turbulence intensity and its variability over New Mexico and Japan, Proceedings, 
9th International Workshop on Technical and Scientific Aspects of MST Radar, Toulouse, 237-240, SCOSTEP, Ed. B. Edwards, 2000.

Tsuda, T., Sato, T., Hirose, K., Fukao, S., and Kato, S., MU radar observations of the sapect sensitivity of backscattered VHF echo power in the troposphere and lower stratosphere, Radio Sci., 21, 971-980, 1986.

Tsuda, T., VanZandt, T. E., and Saito, J., Zenith-angle dependence of VHF specular reflection echoes in the lower atmosphere, J. Atmos. Solar-Terr. Phys., 59, 761-775, 1997a.

Tsuda, T., Gordon, W. E., and Saito, H., Azimuth angle variations of specular reflection echoes in the lower atmosphere observed with the MU radar, J. Atmos. Solar-Terr. Phys., 59, 777-784,
$1997 b$.

Worthington, R. M. and Thomas, L., Long-period unstable gravitywaves and associated VHF radar echoes, Ann. Geophysicae, 15, 813-822, 1997.

Worthington, R. M., Palmer, R. D., and Fukao, S., An investigation of tilted aspect-sensitive scatterers in the lower atmosphere using the MU and Aberystwyth VHF radars, Radio Sci., 34, 413-426, 1999.

Worthington, R. M., Palmer, R. D., Fukao, S., Yamamoto, M., and Astin, I., Rapid variations in echo power maps of VHF radar backscatter from the lower atmosphere, J. Atmos. Solar-Terr. Phys., 62, 573-581, 2000. 